\title{
Study on Information Release of Urban Rail Transit Passenger Flow Induced under Emergencies
}

\author{
Han Tang ${ }^{1}$, Jie $\mathrm{Xu}^{2, *}$, Jianyuan Guo ${ }^{1}$ \\ ${ }^{1}$ School of Traffic and Transportation, Beijing Jiaotong University, Beijing, China \\ ${ }^{2}$ State Key Laboratory of Rail Traffic Control and Safety, Beijing Jiaotong University, Beijing, China \\ 13120894@bjtu.edu.cn,jxu1@bjtu.deu.cn,jyguo@bjtu.edu.cn
}

\begin{abstract}
Urban rail Transit system is a dynamic, uncertain and complex system, it will seriously affect the traffic capacity of road network, and the convenience of people's travel in the event of emergencies. So the timely and accurate release of information is an important guarantee of the safe and efficient operation of rail transit. At first, the problems, which existed in the process of releasing information of the rail transit passenger flow guidance under emergencies are analyzed, Then the functional structure of the system is designed. Based on the structure, this paper does a research on the process of the information release, and puts forward the calculation method in a general influencing scope. Combined with examples, the detail processes of the information release of urban rail transit passenger flow under emergencies are illustrated.
\end{abstract}

Keywords-Urban Rail Transit; Emergency; Passenger flow induced; Information Release

\section{INTRODUCTION}

With the rapid development of urban rail transit, not only the subway network structure becomes more complex, but also the natural disasters, accidents and other emergencies are also increasing, which has brought a series of traffic safety problems at the same time. It has huge influence on the safe and efficient operation of the urban rail transit.

When emergencies happened in urban rail transit, the information release of traffic guidance for traveler can provide timely traffic information, improving the path choice, shortening the travel time and easing traffic congestion. There have been many studies at home and abroad about the information release of traffic induced. The commonly used research methods are field investigation [1], computer model [2], analytical evaluation [3][6]. The research content mainly concentrated in the content and key technology of the induction information release [4][5], the influence on traveler behavior, including changes in the speed, path, pattern, and the influence of induction information release location for traffic flow [7][8].

At present, the research on emergency information release is more about road. Relative to the road traffic areas, the urban rail transit has its own typical characteristics, like good planning, block station and the fixed line.

For the urban rail transit system is an open, uncertain, complex dynamic system, and has a unique public properties, it's vulnerable to the impact of the incidents. What's more, emergency is sudden, severe and urgent, and it often has dynamic change. With the development of the event, its influence changes, so as to the scope and contents of information. Then the system can do the corresponding adjustment to monitor the event timely and to provide accurate information for passengers, who can adjust their travel path and way according to the changes. But current information release system isn't meet these requirements, so it's necessary to establish a diversified, streamline emergency dynamic information release system to pass event information to passengers timely and accurately, and to reduce the effect of emergency on people.

\section{THE INFORMATION RELEASE SYSTEM OF URBAN RAIL TRANSIT PASSENGER FLOW INDUCED UNDER EMERGENCY}

\section{A. General design of system}

According to the current problems existing in the urban rail transit, build the information release system of passenger flow induced of the urban rail transit. The system function structure design as follows:

- Operation Terminal. Operation Terminal is used by the staff, and provides the function interface that information related to event entry, editing and publishing operation, release information of dynamic monitoring and historical information to check.

- Event analysis server. According to the event information which the staff input, Event analysis server calculates the events' influence scope, and complete the release scope and mode matching.

- The template and configuration management server. Template and configuration management server is mainly responsible for inducing text templates and graphics template management, the commonly used configuration management which information release terminal equipment takes up.

- The information release server. According to the calculated content from Event analysis server as well as the template and the configuration manager transmit to the template information, the information 
release server fast match generated release information, and control information release process.

- Data management server. Data management server manage and maintain the basic data and dynamic data of the information release, including basic network, schedules, network operation, etc. It provides basic data for Operate Terminal, event analysis server, template and configuration management server, and information release server, and maintains the data changes..

\section{B. Urban rail transit emergency passenger flow induced information release process}

As the track traffic incident is sudden and uncertain, so the method of the emergency information release of induced passenger flow is different from other types of events. According to the characteristics of the emergency, the system processes and releases the emergency information as the different steps, which has realized the routing of induced passenger flow information.

The emergency information release process is shown in figure 1 .

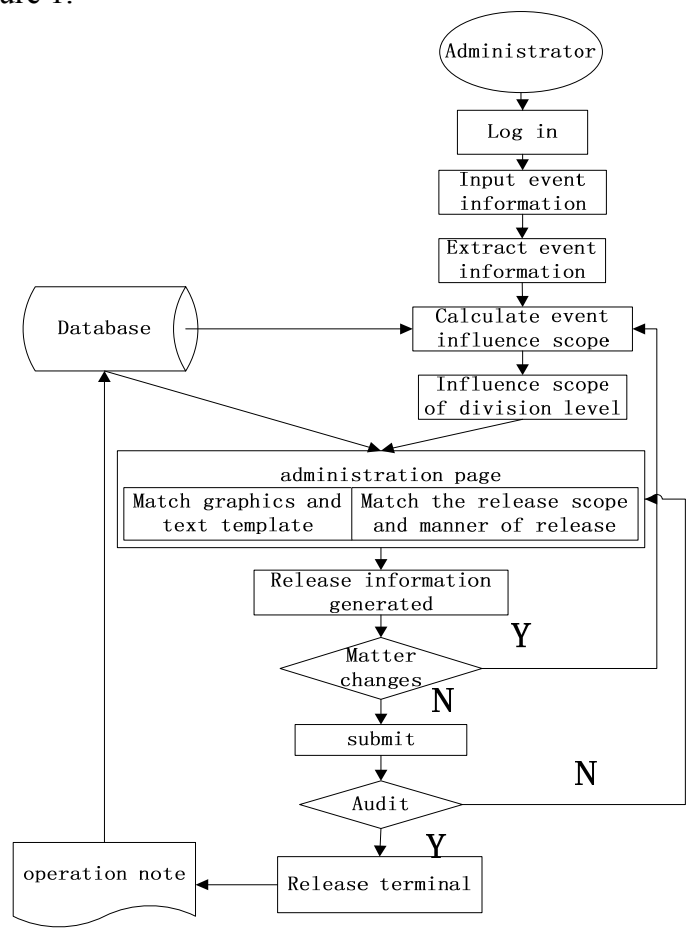

Figure 1. The flow chart of the emergency information release

Emergency information release steps:

- After the emergency, the administrator login system by manipulating the terminal equipment to enter information.

- Event management technology is employed to extract incident type, location, time and other information .

- According to the emergency attributes, using historical data and network based data, calculated the influence of emergencies, level and scope of influence.

- According to calculate the classification of the scope of the events and the template information in the database, to appropriate graphics, text template match event, and determine the scope of the information release and release, publishing information generated.

- According to the event development, and continuously match event development level and development condition, the influence of real-time computing events and hierarchy of the range, and then update the text and graphics image template, generate real-time event information released to passengers.

- Superior staff audit the release of the information, if pass, then the generated event information will publish to the specified terminal as the specified time, and to control the whole publishing process; Not pass, then return to management page to match the template.

- All operating records are kept in the database, for later queries.

\section{Emergency relevant calculation}

In this paper, on the basis of the duration it deduces the influence scope, the calculation method of information release rate and release time, specific algorithm steps are as follows:

- Assuming that the site of the incident is the station , the duration of the incident is $T$, according the literature [8]

$$
T=t_{d}+t_{r}+t_{c}+t_{R}
$$

Where $t_{d}$ is discovery time, $t_{r}$ is response time, $t_{c}$ is clearing time, $t_{R}$ is Recovery time.

- To determine the shortest travel time $T^{\prime}$ from the station $O$ to any point $D$ on Road network

$$
\left\{\begin{array}{l}
T^{\prime}=\min \left(T_{n}^{\prime}\right) \\
T_{n}^{\prime}=t_{n 1}+t_{n 2}+t_{n 3}
\end{array}, n \geq 1\right.
$$

Where $n$ is the number of the path from $O$ to $D, t_{n 1}, t_{n 2}, t_{n 3}$ respectively mean the train operating time, the train average stopping time, passenger average transfer time. There, passenger average transfer time include the transfer line and transfer waiting time.

- Compute the influence scope $G$ of emergency. Here, the duration $T$ of the events is the event influence time, if $T^{\prime} \leq T$, so $D \in G$.That is, within the network incident can only affect the traveler who 
may arrive at the station within the scope of influence time.

- Divide the hierarchy of the influence scope $G$ of emergency. The event time is divided into three stages, expression by $T_{i}, i=1,2,3$ and $T_{i} \leq T_{i+1}$. Result from the influence scope $G_{i}$ of each time period. According to the distance from the stations and lines on network to the site of the incident, $G$ is carried on the hierarchy, there is

$$
G_{i}{ }^{\prime}=G_{i}-G_{i-1}\left(G_{0}=0\right), i=1,2,3
$$

- Where $G_{1}{ }^{\prime}$ is the serious influence area, $G_{2}{ }^{\prime}$ is the general influence area, $G_{3}{ }^{\prime}$ is the probable influence area, $T_{i}$ obtained by manually or by empirical data in the database.

- Determine stations' information release speed and the release time. According to [10], information release speed is measured in time, expression by $t$, according to the following formula.

$$
t=\left\{\begin{array}{l}
t_{\alpha}+t_{\beta}, 0 \leq t_{\alpha}, t_{\beta}<\infty \\
\operatorname{Max}\left(\left(t_{\alpha}+t_{\beta}\right), t_{\mu}\right), 0 \leq t_{\alpha}, t_{\beta}, t_{\mu}<\infty
\end{array}\right.
$$

where $t_{\infty}$ is the original data acquisition cycle, $t_{\beta}$ is information processing time, $t_{\mu}$ is the emergency information release cycles. For the formula (4), if $t_{\infty}+t_{\beta}>t_{\mu}, t=t_{\infty}+t_{\beta}$, so we think this is an invalid release, i.e., release information time is greater than the time needed for emergency disposal:

- Hypothesis the moment of incident is $t^{\prime}$ at station $O$, the information release time of the various stations is

$$
t^{\prime} \sim\left(t^{\prime}+\left(T-T^{\prime}\right)\right), T-T^{\prime} \geq 0
$$

\section{Emergency induced passenger flow information release instance}

Construction of road network structure as shown in figure 2, assuming Line fault occurred at $C D$, time of fault is $14: 20$, the time of duration is $15 \mathrm{~min}$, the travel time between two adjacent is 5min, the information release implementation steps as follows:

- The administrator login system, and choose events for the line fault through the human-computer interaction interface in terminal. Fault line is $C D$, and influence of time is $10 \mathrm{~min}$.

- By the event influence range cell, it is engaged in the starting points, calculating the range of influence events.

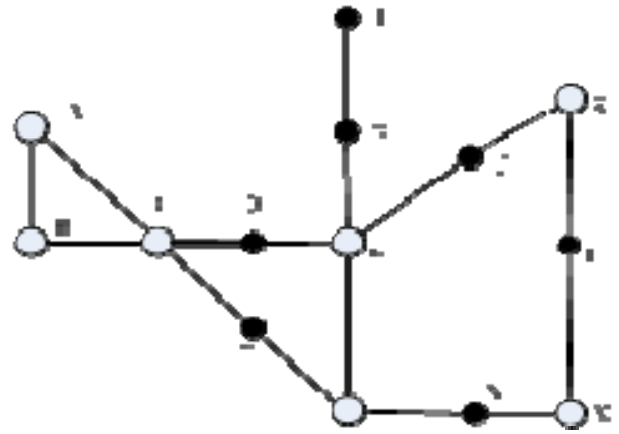

Figure 2. .Road network structure

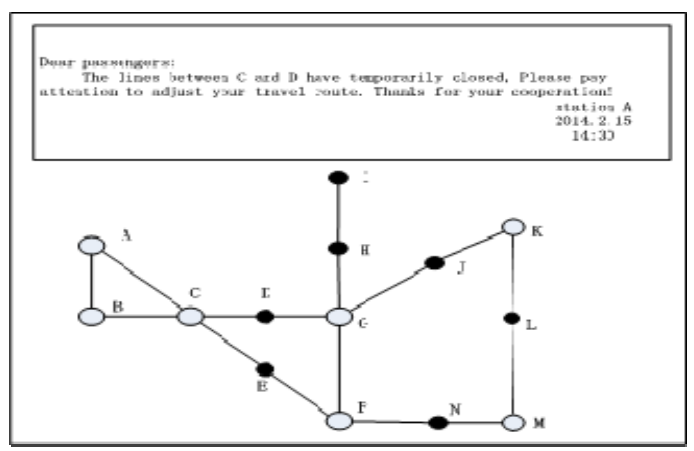

Figure 3 A station display screen

The scope of collection $G=\{A B, A C, B C, C D, C E, E F$, $F N, D G, G F, G H, H I, G J, J K\}$

$N M, K L, L M$ is the unaffected area. To $5 \mathrm{~min}$ to divide the scope of events, and according to the severity of dividing area below:

the serious influence $G_{1}{ }^{\prime}=\{A C, B C, C D, C E, D G\}$

the general influence area $G_{2}{ }^{\prime}=\{A B, E F, G F, G H, G J\}$

the probable influence area $G_{3}{ }^{\prime}=\{H I, J K, F N\}$

- Release time is calculated in PIS terminal stations:, $C$ and $D$ is 14:20-14:35; $A, B, E$ and $G$ is $14: 20-14: 30$; $H, J$ and $F$ is $14: 20-14: 25$, the rest of the stations don't have to publish the information. Release template configuration unit depend on the type of events to choose a template type and match the template elements, matching the templates as different areas and getting the text content to release. Then logic and semantic are checked to ensure content logically and semantically correct, then submit it.

- Staff login system audit. After the approval, release information to PIS terminal equipment according to the set moment. At 14:30, the station $A$ display images as shown in figure 3 .

- According to the change of events and disposal measures, generate new content and update the information quickly.

- After the incident, the release terminal display daily published content. 


\section{CONCLUSION}

In order to ensure the safety and efficient operation of urban rail traffic, it should establish a diversified, streamline emergency dynamic information publishing system. In this system, according to the relative event attributes, it can automatically generate the time and space of the terminal equipment when releasing information; according to the template and the basic configuration, it can quickly generate the text and graphics templates. This study, which puts forward this kind of system has a certain value and significance for it supports the control of process and the dynamic management when information releases, so that the standardization and the efficiency are improved.

\section{REFERENCES}

[1] Adler J L, Blue V J. Toward the design of intelligent traveler information systems[J] . Transportation Research Part C, 1998, 3 (6): 157- 172.

[2] Reddy P D V G, Yang H, Vaughn K M, et al. Design of an artificial simulator for analyzing route choice behavior in the presence of information system[J]. Transportation Research Part C, 1995, 8 (22): $119-147$.
[3] Linda Ng Boyle, Mannering F. Impact of traveler advisory systems on driving speed: some new evidence [J]. Transportation Research Part C, 2004, 5 (12): 57- 72.

[4] Wang Hui, Jin Zhongcun, Wu Wei. The development of GPS navigation system based on dynamic traffic information research[J]. Communications Standardization, 2006 (1): 33- 35..

[5] Yin Tao, Liang Qian. The ATMS system for traffic information research[ J]. Road, 2009, 3(3): 87- 91.

[6] Susan Kenyon, Glenn Lyons. The value o f integrated multimodal traveler information and its potential contribution to mode change $[\mathrm{J}]$. Transportation Research Part F, 2003(6) : 1- 23.

[7] Thakuriah, P., Sen, A. Quality of information given by advanced traveler information systems[J]. Transportation Research Part C, 1996,10 (4): 249-266.

[8] Hato E, Taniguchib M, Sugiec Y, et al. Incorporating an information acquisition process into a route choice model with multiple information sources[J] . Transport at ion Research Part C,1999, 5(7): 109- 129.

[9] Highway capacity manual (HCM)(2000). Transportation Research Board, National Research Council,Washington, D.C.

[10] Mao Baohua, Liu Mingjun. Rail transit network operation organization theory and key technology[M]. Beijing: Sciences Press, 2011, 119-129.

[11] http://www.docin.com/p-203376358.html\#documentinfo 